\title{
Detection of the SARS-CoV-2 Nucleocaspid Protein (NP) Using Immunohistochemistry
}

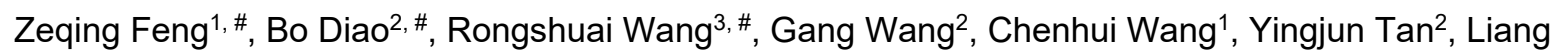

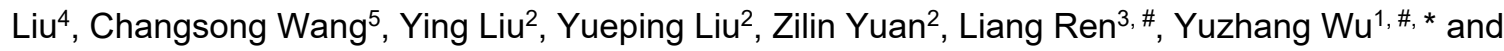

\author{
Yongwen Chen ${ }^{1, \#, *}$
}

${ }^{1}$ Institute of Immunology, PLA, Third Military Medical University, Chongqing, 400038, People's Republic of China; ${ }^{2}$ Department of Medical Laboratory Center, General Hospital of Central Theater Command, Wuhan, Hubei province, 430015, People's Republic of China; ${ }^{3}$ Hubei Chongxin Judicial Expertise Center, Wuhan, Hubei province, 430415, People's Republic of China; ${ }^{4}$ Department of Forensic Medicine, Tongji Medical College, Huazhong University of Science and Technology, No. 13 Hangkong Road, Wuhan, Hubei province, 430030, People's Republic of China; ${ }^{5}$ Department of Pathology, 989 th Hospital of PLA, Luoyang, 471000, Henan Province, People's Republic of China \#Contributed equally to this work

*For correspondence: wuyuzhang@tmmu.edu.cn; yongwench@163.com

[Abstract] While lymphocytopenia is a common characteristic of patients infected by the novel severe acute respiratory syndrome coronavirus 2 (SARS-CoV-2), the mechanisms responsible for this depletion are unclear. With the tissue samples of the spleens and lymph nodes (LNs) from six cases, immunohistochemistry demonstrated ACE2 (angiotensin-converting enzyme 2), the potential receptor of SARS-CoV-2, expresses on tissue-resident CD169+ macrophages in spleens and LNs.

Keywords: SARS-CoV-2, ACE2, Spleen, Lymph nodes, Immunohistochemistry

[Background] In December 2019, clusters of patients with pneumonia of unknown etiology were reported by the local health facilities in Wuhan, Hubei Province, China (Li et al., 2020; Zhu et al., 2020). In January 2020, the causative agent of mysterious pneumonia was identified as a novel coronavirus named as 2019 novel coronavirus (2019-nCoV) by the World Health Organization (WHO). This novel coronavirus was renamed as the novel severe acute respiratory syndrome coronavirus 2 (SARS-CoV-2), and the consequent disease was designated as coronavirus disease 2019 (COVID-19) (Lu et al., 2020; Xu et al., 2020). According to the daily report of the WHO, the epidemic of SARS-CoV-2 has so far caused 81961 laboratory confirmed cases and 3293 death in China. Meanwhile, the number of confirmed COVID-19 and fatalities around the world are 465315 and 21031, respectively, by $26^{\text {th }}$ March 2020 (World Health Organization, 2020).

The importance of the secondary lymphoid organs, including spleen and lymph nodes (LNs), for resistance against infection is well established. Tissue-resident macrophages positioned in the splenic marginal zone (MZ) that are among the first cell types to encounter invading pathogens (Aichele et al., 2003; Mebius and Kraal, 2005). Similarly, the resident macrophages of the subcapsular sinus and hilar lymph nodes have been shown to play a protective role against viral 
infections by capturing viral particles (Junt et al., 2007; Tamburini et al., 2014). These macrophages present viral antigens to activate specific $\mathrm{T}$ cells, to induce activation, clonal proliferation, and subsequent killing of infected target cells through secretion of perforin, granzyme $B$, and interferony etc (Backer et al., 2010; Bernhard et al., 2015; van Dinther et al., 2018). Moreover, these activated $T$ cells also egress from spleen and LNs into blood circulation and play immune surveillance roles (Gebhardt et al., 2013). Unfortunately, our previous work has shown that lymphocytopenia is prevalent in COVID-19 patients, especially in aged and critically ill cases, suggesting that the immune system from COVID-19 patients might be neutralized by infection (Diao et al., 2020).

Therefore, understanding the destinies of lymphocytes in secondary lymphoid organs is critical for understanding SARS-CoV-2 viral infection, and the identification of methods to boost lymphocytes and enhance the host immunity in vivo.

In this study, the tissue samples of spleens and LNs are from six COVID-19 patients. Viral nucleocapsid protein (NP) antigen, cell apoptosis and proinflammatory cytokine expression were measured by immunohistochemistry. And the protocol is detailed below.

\section{Materials and Reagents}

1. Glass slides

2. Paraffin-embedded tissue blocks

3. Poly-L-lysine

4. Hydrogen peroxidase

5. Methanol

6. BSA

7. PBS

8. Nonidet P-40

9. Hematoxylin

10. Ethanol

11. The Vecta-stain $A B C$ kit (Vector Laboratories, San Diego, CA, USA)

12. DAB Elite kit (DAKO, Copenhagen, Denmark, catalog number: K3465)

13. Antibodies anti-SARS-CoV-2 nucleocaspid protein (NP) antibodies (1:100, rabbit lgG) (Sino Biological, Beijing, clone ID: 019) anti-ACE2 (1:100, rabbit IgG) (Sino Biological, clone ID: 10108-RP01) anti-CD68 (1:100, mouse IgG1) (BIO-RAD, Clone ID:KP1) anti-CD169 (1:100, mouse IgG1) (Biolegend, Clone ID:7-239) anti-B220 (1:100, mouse IgG1) (BIO-RAD, Clone ID:123C3) anti-Fas (1:100, mouse IgG) (Thermo fisher, ID:48095942) anti-FasL (1:100, rabbit IgG) (Santa Cruz, catalog number: sc-834) anti-IL-6 (1:100, mouse IgG2b) (Santa Cruz, catalog number: sc-130326) 
rabbit-isotype antibody controls (1:100) (Dako)

14. $10 \mathrm{mM}$ citrate buffer $(\mathrm{pH}=6.0)$ (see Recipes)

15. $0.5 \%$ hydrogen peroxidase $\left(\mathrm{H}_{2} \mathrm{O}_{2}\right)$ (see Recipes)

\section{Equipment}

1. Light microscope (Zeiss Axioplan 2)

\section{Procedure}

In the protocol, tissue samples of the spleens and LNs from six COVID-19 patients were collected and used upon the approval of the National Health Commission of China and Ethics Commission of General Hospital of Central Theatre Command and Jinyintan Hospital.

1. Cut the paraffin-embedded tissue blocks into $2-3 \mu \mathrm{m}$ sections.

2. Mount them on poly-L-lysine-charged glass slides.

3. De-waxe and rehydrate the sections

4. Add the sections in $10 \mathrm{mM}$ citrate buffer $(\mathrm{pH} \mathrm{6.0)}$ and perform the antigen retrieval with microwave.

5. Incubate with a solution of $0.5 \% \mathrm{H}_{2} \mathrm{O}_{2}$ in $50 \%$ methanol for $1 \mathrm{~h}$ to block the endogenous peroxidase activity.

6. Incubate in $3 \%$ BSA plus $0.1 \%$ Nonidet P-40 in PBS for $1 \mathrm{~h}$ at room temperature. So that the nonspecific binding is blocked.

7. Dilute the primary antibodies with $1 \%$ BSA.

Notes: The primary antibodies consist of anti-SARS-CoV-2 nucleocaspid protein (NP) antibodies, anti-ACE2, anti-CD68, anti-CD169, anti-B220, anti-Fas, anti-FasL, anti-IL-6 or rabbit-isotype antibody controls (1:100; Dako).

8. Incubate the sections in the diluted primary antibodies overnight at $4{ }^{\circ} \mathrm{C}$.

9. Wash the sections.

10. Incubate the sections with the corresponding secondary antibodies for $1 \mathrm{~h}$ at room temperature.

11. Perform the avidin- biotin complex method with the Vecta-stain $A B C$ kit. Detailed procedures are followed with the manufacturer's instructions.

12. Incubate the sections with isotype-matched concentration-matched immunoglobulin. Note: No primary antibodies are used as isotype controls.

13. Visualize the peroxidase activity with the DAB Elite kit.

Note: Brown coloration of tissues represents positive staining.

14. Counterstain the sections with hematoxylin.

15. Dehydrate the sections through an ethanol series to xylene and mount them.

16. View the sample sections with a light microscope (Figure 1). 

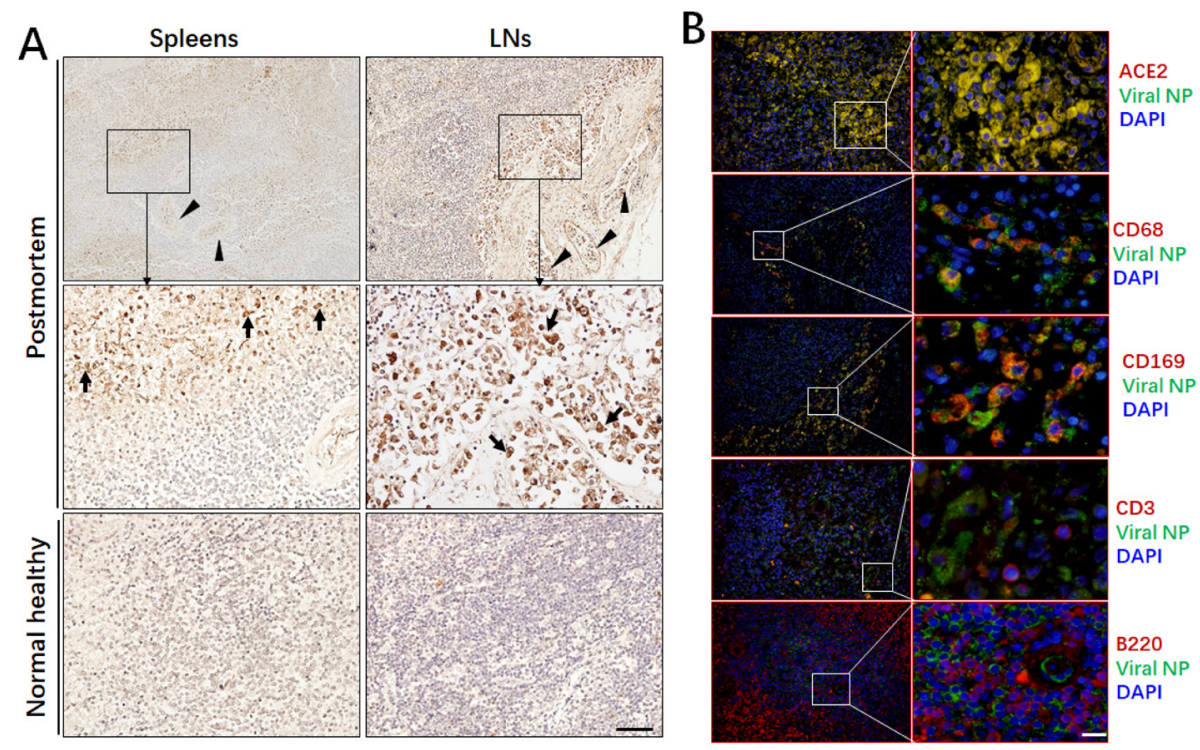

Figure 1. Immunohistochemistry analyzed SARS-CoV-2 NP antigen in spleen and LN tissues. A. The expression of viral NP antigen was detected by immunohistochemistry in spleen and LN tissues from COVID-19 patients undergoing postmortem examination and age- match normal healthy controls. B. Immunofluorescent double staining analyzed viral NP antigen expression. Arrow indicated viral NP positive cells. Scale bar= $100 \mu \mathrm{M}$. (Chen et al., 2020)

\section{$\underline{\text { Recipes }}$}

1. $10 \mathrm{mM}$ citrate buffer $(\mathrm{pH}=6.0)$

2. $0.5 \%$ hydrogen peroxidase $\left(\mathrm{H}_{2} \mathrm{O}_{2}\right)$

\section{Acknowledgments}

Yuzhang $\mathrm{Wu}$, and Yongwen Chen were involved in the final development of the project and manuscript preparation; Zilin Yuan, Chenghui Wang and Zeqing Feng analyzed the data; Bo Diao, Yin Liu, Gang Wang, Yinjun Tan and Yueping Liu did H\&E staining and immunohistochemistry; Changsong Wang evaluated H\&E and immunohistochemistry results; Liang Liu, Rongshuai Wang and Liang Ren provided autopsies and analyzed H\&E staining.

\section{Competing interests}

The authors declare no financial or commercial conflict of interest.

\section{Ethics}

This study was approved by the National Health Commission of China and Ethics Commission of 
General Hospital of Central Theatre Command and Jinyintan Hospital.

\section{References}

1. Aichele, P., Zinke, J., Grode, L., Schwendener, R. A., Kaufmann, S. H. and Seiler, P. (2003). Macrophages of the splenic marginal zone are essential for trapping of blood-borne particulate antigen but dispensable for induction of specific T cell responses. J Immunol 171(3): 1148-1155.

2. Backer, R., Schwandt, T., Greuter, M., Oosting, M., Jungerkes, F., Tuting, T., Boon, L., O'Toole, T., Kraal, G., Limmer, A. and den Haan, J. M. (2010). Effective collaboration between marginal metallophilic macrophages and $\mathrm{CD} 8^{+}$dendritic cells in the generation of cytotoxic $\mathrm{T}$ cells. Proc Natl Acad Sci U S A 107(1): 216-221.

3. Bernhard, C. A., Ried, C., Kochanek, S. and Brocker, T. (2015). $\underline{\mathrm{CD}_{169^{+}} \text {macrophages are }}$ sufficient for priming of CTLs with specificities left out by cross-priming dendritic cells. Proc Natl Acad Sci U S A 112(17): 5461-5466.

4. Chen, Y. W., Feng, Z. Q., Diao, B., Wang, R. S., Wang, G., Wang, C. H., Tan, Y. J., Liu, L., Wang, C. S., Liu, Y., Liu, Y. P., Yuan, Z. L., Ren, L. and Wu, Y. Z. (2020). The novel severe acute respiratory syndrome coronavirus 2 (SARS-COV-2) directly decimates human spleens and lymph nodes. medRxiv: 2020.2003.2027.20045427.

5. Diao, B., Wang, C. S., Tan, Y. J., Chen, Y. W., Liu, Y., Ning, L. F., Chen, L., Li, M., Liu, Y. P., Wang, G., Yuan, Z. L., Feng, Z. Q., Wu, Y. Z. and Chen, Y. W. (2020). Reduction and functional exhaustion of $T$ cells in patients with coronavirus disease 2019 (COVID-19). medRxiv: 2020.2002.2018.20024364.

6. Gebhardt, T., Mueller, S. N., Heath, W. R. and Carbone, F. R. (2013). Peripheral tissue surveillance and residency by memory T cells. Trends Immunol 34(1): 27-32.

7. Junt, T., Moseman, E. A., lannacone, M., Massberg, S., Lang, P. A., Boes, M., Fink, K., Henrickson, S. E., Shayakhmetov, D. M., Di Paolo, N. C., van Rooijen, N., Mempel, T. R., Whelan, S. P. and von Andrian, U. H. (2007). Subcapsular sinus macrophages in lymph nodes clear lymph-borne viruses and present them to antiviral B cells. Nature 450(7166): 110-114.

8. Li, Q., Guan, X., Wu, P., Wang, X., Zhou, L., Tong, Y., Ren, R., Leung, K. S. M., Lau, E. H. Y., Wong, J. Y., Xing, X., Xiang, N., Wu, Y., Li, C., Chen, Q., Li, D., Liu, T., Zhao, J., Liu, M., Tu, W., Chen, C., Jin, L., Yang, R., Wang, Q., Zhou, S., Wang, R., Liu, H., Luo, Y., Liu, Y., Shao, G., Li, H., Tao, Z., Yang, Y., Deng, Z., Liu, B., Ma, Z., Zhang, Y., Shi, G., Lam, T. T. Y., Wu, J. T., Gao, G. F., Cowling, B. J., Yang, B., Leung, G. M. and Feng, Z. (2020). Early transmission dynamics in wuhan, china, of novel coronavirus-infected pneumonia. N Engl J Med 382(13): 1199-1207.

9. Lu, R., Zhao, X., Li, J., Niu, P., Yang, B., Wu, H., Wang, W., Song, H., Huang, B., Zhu, N., Bi, Y., Ma, X., Zhan, F., Wang, L., Hu, T., Zhou, H., Hu, Z., Zhou, W., Zhao, L., Chen, J., Meng, Y., Wang, J., Lin, Y., Yuan, J., Xie, Z., Ma, J., Liu, W. J., Wang, D., Xu, W., Holmes, E. C., Gao, G. F., Wu, G., Chen, W., Shi, W. and Tan, W. (2020). Genomic characterisation and epidemiology 
of 2019 novel coronavirus: implications for virus origins and receptor binding. Lancet 395(10224): 565-574.

10. Mebius, R. E. and Kraal, G. (2005). Structure and function of the spleen. Nat Rev Immunol 5(8): 606-616.

11. Tamburini, B. A., Burchill, M. A. and Kedl, R. M. (2014). Antigen capture and archiving by lymphatic endothelial cells following vaccination or viral infection. Nat Commun 5: 3989.

12. van Dinther, D., Veninga, H., Iborra, S., Borg, E. G. F., Hoogterp, L., Olesek, K., Beijer, M. R., Schetters, S. T. T., Kalay, H., Garcia-Vallejo, J. J., Franken, K. L., Cham, L. B., Lang, K. S., van Kooyk, Y., Sancho, D., Crocker, P. R. and den Haan, J. M. M. (2018). Functional CD169 on macrophages mediates interaction with dendritic cells for $\mathrm{CD} 8^{+} \mathrm{T}$ cell cross-priming. Cell Rep 22(6): 1484-1495.

13. World Health Organization. (2020). Novel cornoavirus (COVID-19) situation. (Date accessed: March 27, 2020).

14. Xu, X. W., Wu, X. X., Jiang, X. G., Xu, K. J., Ying, L. J., Ma, C. L., Li, S. B., Wang, H. Y., Zhang, S., Gao, H. N., Sheng, J. F., Cai, H. L., Qu, Y. Q. and Li, L. J. (2020). Clinical findings in a group of patients infected with the 2019 novel coronavirus (SARS-Cov-2) outside of Wuhan, China: retrospective case series. BMJ 368: m606.

15. Zhu, N., Zhang, D., Wang, W., Li, X., Yang, B., Song, J., Zhao, X., Huang, B., Shi, W., Lu, R., Niu, P., Zhan, F., Ma, X., Wang, D., Xu, W., Wu, G., Gao, G. F., Tan, W., China Novel Coronavirus, I. and Research, T. (2020). A novel coronavirus from patients with pneumonia in China, 2019. N Engl J Med 382(8): 727-733. 